\title{
A REVIEW OF SPACE HELIOSEISMOLOGY
}

\author{
T. TOUTAIN
}

Observatoire de la Côte d'Azur

BP 4229, F-06304 Nice cedex 04

\section{Introduction}

The helioseismology started in 1960 with the discovery of oscillations of the velocity field on the solar surface by Leighton, 1960. The mechanism producing these oscillations remained unexplained for the next ten years until Ulrich, 1970, and independently Leibacher and Stein, 1971, demonstrated that acoustic waves trapped beneath the solar surface could generate such oscillations. According to that theory the Sun should oscillate globally and not only locally as seen in the pionnering observations. First Deubner, 1975, and then Claverie et al., 1979, and Grec et al., 1980, demonstrated the existence of global oscillations of intermediate and low degrees. These observations analysed in terms of $k-\omega$ diagram and power spectrum led to the famous p-modes ridges and spectrum lines which are at the base of any inference of the solar structure. All these observations where carried out from the ground. Nevertheless ground-based observations at that time (early eighties) were suffering some limitations which could be over taken from space. This is the reason why the eighties saw the intensive development both of ground-based networks and space missions for helioseismology resulting in 1995 in the existence of four networks: BISON, IRIS, GONG and TON (see Pallé, 1996) and the launch of the SOHO satellite (Domingo et al., 1995). In the next section I review the limitations of ground-based observations and those of space missions. In section 3, I describe in a chronological order the past space helioseismology missions (from 1980 to 1994). Furthermore I discuss, in section 4, the present status of space helioseismology with $\mathrm{SOHO}$ and finally in section 5 the co-ordination of space missions and ground-based solar observations. In the last section the future and the prospects of space helioseismology are reviewed. 


\section{Limitations in the early eighties}

At the end of the seventies and the beginning of the eighties the observations of the solar oscillations were suffering from mainly three limitations.

\subsection{TIME COVERAGE OF OBSERVATIONS}

At that time, the ground-based instruments observed the Sun separately with interruption during night-time and bad weather conditions. In terms of Fourier power spectrum the regular gaps in the time series due to night and day cycles introduce side bands at $11.57 \mu \mathrm{Hz}$ and other spurious peaks in the case of irregular interruptions. All these peaks make the identification of modes more difficult and the determination of their frequencies less accurate. From simulations (Noyes and Rhodes, 1984), we know that gap filling methods are able to recover the original power spectrum for duty cycles better than $80 \%$, especially if the gaps are irregularly spaced in time. There was an attempt to bypass this limitation by Grec et al., 1980) who were able to observe the Sun continuously during five days from the South Pole. Though it was a great improvement in term of continuity, it showed the second limitation of experiments in helioseismology; namely the short-time duration of observations.

\subsection{DURATION OF OBSERVATIONS}

Long-uninterrupted observations can improve the determination of frequencies in two different ways. First by increasing the frequency resolution and second by increasing the signal-to-noise ratio for modes having lifetime longer than the observation duration. For example the 5 days of continuous observations obtained from the South Pole allow a frequency resolution of only $2.3 \mu \mathrm{Hz}$ which is not enough to resolve neither the rotational splitting of the modes $(0.45 \mu \mathrm{Hz})$ nor the lines of modes below $2 \mathrm{mHz}$ which have lifetime of the order of a month. In addition the increase of solar noise with decreasing frequency makes detection of low frequency modes more difficult. This effect is due to granulation and supergranulation noise and is emphasized with ground-based observations because of the influence of the Earth's atmosphere.

\subsection{INFLUENCE OF THE EARTH'S ATMOSPHERE}

It is obvious that the motion of the Earth's atmosphere due to turbulence strongly affect the observations of solar oscillations. Low-degree mode observations are affected by an extra noise at low-frequencies whereas highdegree mode observations are affected by the loss of coherency on the solar 
disk. This produces a leakage of the high-degree modes and a conversion of the steady velocity flows in apparent time-varying signals. Simulations of seeing effect on $\mathrm{p}$ modes ridges (Noyes and Rhodes, 1984) show that modes for $l>300$ are embedded in the noise already with a 4" seeing which is a typical seeing under turbulent conditions.

The present status of observations in helioseismology is different. Groundbased networks do not suffer from the two first limitations though the last one is still there. The only way to avoid the influence of the Earth's atmosphere is to fly space missions. Nevertheless space missions have also some disadvantages like their cost and the impossibility to repair any instrumental defect. For these reasons the first observations of solar oscillations from space were not made with a dedicated instrument.

\section{Past of space helioseismology (1980-1994)}

From an historical point of view and though it was designed for solar constant measurements and not for helioseismology, ACRIM was the first instrument to observe solar oscillations from space.

\subsection{ACRIM}

The ACRIM (Active Cavity Radiometer Irradiance Monitor) instrument was launched the $14^{\text {th }}$ of February 1980 aboard the SMM (Solar Maximum Mission) satellite of NASA. The orbit consisted in a $550-\mathrm{km}$, low-inclination Earth orbit. The corresponding orbital period was $95 \mathrm{mn}$ with a night of $35 \mathrm{mn}$. This kind of regular data gaps due to night-time generate in power spectrum side bands at $+/-170 \mu \mathrm{Hz}$. The instrument was composed of 3 independent cavity radiometers operated differentially against a common thermally massive heat sink (see Willson, 1979 for more details). As explained before this instrument was designed for total irradiance measurements and therefore sensitive to UV, visible and IR radiation. Its relative accuracy was far better then $0.1 \%$. That is to say good enough to detect solar $\mathrm{p}$ modes in integrated sunlight measurements. At that time, none of the attempts from ground to detect $\mathrm{p}$-mode signature in integrated sunlight were successful. Therefore ACRIM is both the first space experiment to detect $\mathrm{p}$ modes and also the first experiment to detect $\mathrm{p}$ modes in the integrated sunlight.

\subsubsection{Scientific outcomes}

The main results from ACRIM as far as helioseismology is concerned (Woodard and Hudson, 1983a,b, Woodard, 1984) were obtained with a time series of 10 months gathered during the first year of mission and before the loss of spacecraft fine-pointing control. The duty cycle of ACRIM during these 


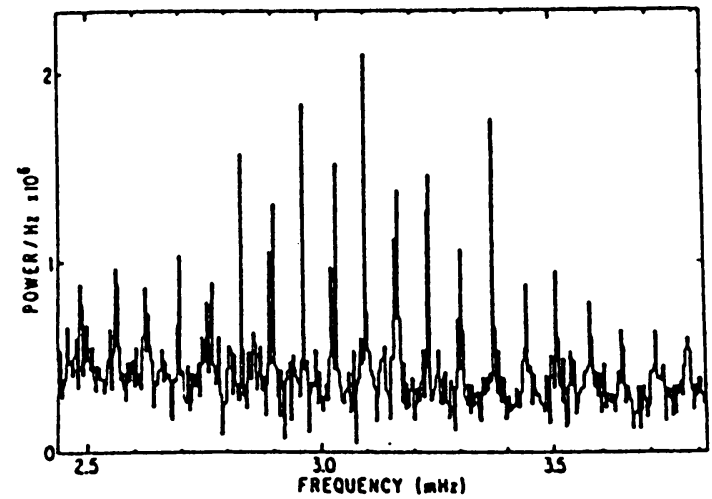

Figure 1. 10-month ACRIM spectrum Woodard, 1984

10 months was about $30 \%$. With this time series was obtained the famous ACRIM p-mode spectrum (fig.1), where p modes of degrees $l=0,1$ and 2 are visible with a signal-to-noise ratio between 1 and 4 in the frequency range of 2.5 to $3.8 \mathrm{mHz}$. The frequency range was upper-limited by the 131 sec shutter-cycle, leading to a Nyquist frequency of $3.815 \mathrm{mHz}$. The quite good signal-to-noise ratio allowed measurement of the p-mode frequencies (with an accuracy of $0.4 \mu \mathrm{Hz}$ ), their linewidths as well as their amplitudes.

\subsection{STRATOSPHERIC BALLOONS}

In 1980 and 1983 were launched two stratospheric balloons by the french space agency (CNES) with aboard a set of three sunphotometers dedicated to the measurements of the spectral solar irradiance (Fröhlich, 1984). As in the case of ACRIM, the instrument was not designed for helioseismology, but its good stability could allow detection of $p$ modes. The concept was quite simple: three interference filters $(368,500$ and $778 \mathrm{~nm})$ at the entrance of the instrument to select thin wavelength bands of the visible continuum and a silicon-diode as detector. The observing times were rather short: 4 and 7 hours respectively.

\subsubsection{Scientific outcomes}

Because of the short duration of the observations it was not possible to identify separate peaks in the power spectra. Nevertheless it was the first time that $\mathrm{p}$ modes were observed at three different wavelengths (red, green and blue). It was therefore possible from the time series (fig.2) to determine an amplitude ratio and a phase shift between colors as well as their frequency-dependence (Fröhlich and van der Raay, 1984). 


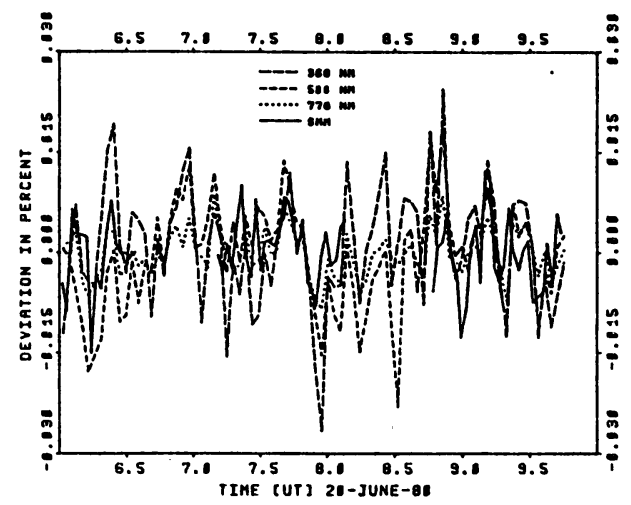

Figure 2. Time series of the three sunphotometer channels and of ACRIM total irradiance (Fröhlich, 1984)

\subsection{IPHIR}

The IPHIR (InterPlanetary Helioseismology by IRradiance measurements) instrument was launched on July 7 and July 12, 1988 aboard the interplanetary russian probes PHOBOS I and PHOBOS II, respectively (Fröhlich et al., 1988). IPHIR can be considered as the first space experiment dedicated to helioseismology. The idea was to take advantage of the long interplanetary flight between the Earth and Mars to observe continuously the Sun. In fact PHOBOS I was lost on September 2, 1988 only 2 months after the launch because of a wrong telecommand sent to the probe. The lifetime of the second probe was quite longer, PHOBOS II was lost in March 27, 1989, far later after the encounter of the probe with the martian satellite, because of a breakdown of the onboard computer. Thus, it allowed the IPHIR experiment aboard PHOBOS II to observe the Sun continuously for more than 160 days. The IPHIR instrument was composed of a triple sunphotometer with 3 interference filters $(335,500$ and $862 \mathrm{~nm})$ and a silicon-diode detector allowing observation of the integrated sunlight at 3 wavelengths of the visible continuum. The relative accuracy of the instrument (better than $1 \mathrm{ppm})$ and the fine pointing provided by a two-axis sun sensor have led to data of good quality though slightly polluted by light diffused inside the instrument. Moreover the blue channel of the photometers never provided good data because of the strong and quick degradation of the blue filter.

\subsubsection{Scientific outcomes}

The long continuous duration of observations with IPHIR yielded to a rather clean power spectrum (fig. 3, Toutain and Fröhlich, 1992) compared to the ACRIM one. The improvement in terms of signal-to-noise ratio was quite significant. This ratio was about 20 for $\mathrm{p}$ modes around $3 \mathrm{mHz}$. P- 


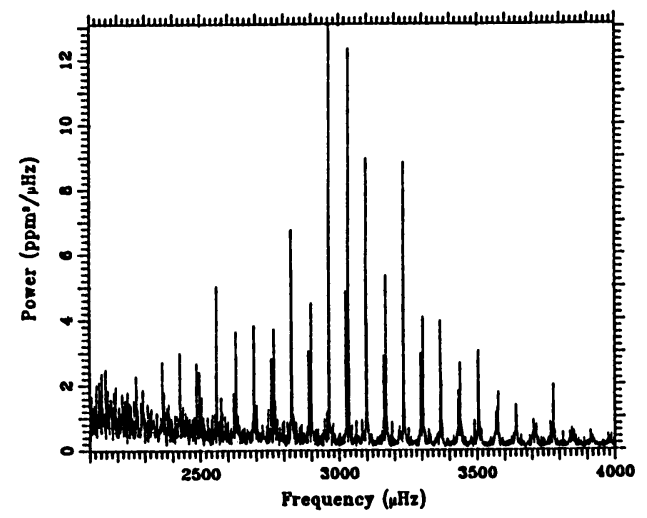

Figure 3. IPHIR green power spectrum (Toutain and Fröhlich, 1992)

mode parameters of $l=0,1$ and 2 modes were measured in a frequency range spanning from 2.4 to $3.8 \mathrm{mHz}$. Frequencies were measured with an accuracy of $0.1 \mu \mathrm{Hz}$. Thanks to the continuous observations it has been demonstrated with IPHIR that p-mode amplitudes change quite strongly with time and in a way which agrees quite well with a model of stochatic excitation (Toutain and Fröhlich, 1992, Baudin et al., 1996). Nowadays it is widely admitted that p-modes are stochastically excited by turbulent convection. With IPHIR, was also measured a mean splitting of $0.56+0.02$ $\mu \mathrm{Hz}$ for modes $\mathrm{l}=1,2$. It has been shown (Toutain and Kosovichev, 1994) that this can be considerably reduced $(0.47+0.04 \mu \mathrm{Hz})$ if a constant splitting is assumed throughout the mode frequency range, thus showing how difficult it is to measure accurately the splitting of low-degree p-modes. Up to now there is still no a complete agreement on the value of the mean splittings of low-degree $\mathrm{p}$ modes between the various experiments which are able to measure these quantities (Appourchaux et al, 1995).

\subsection{SOVA}

Four years after IPHIR was launched on July 31, 1992 aboard the EURECA (EUropean REtrievable CArrier) platform the SOVA (SOlar constant and VAriability) instrument (Crommelynck et al, 1995). The platform was retrieved 1 year later on June 24, 1993 by the space shuttle. This platform was originally planned to be launched in the mid-eigthies but was delayed because of the problems the space shuttle programme encountered. The orbit of EURECA was a 508-km Earth orbit with a period of about $95 \mathrm{mn}$ and a night of $40 \mathrm{mn}$ (Innocenti 1993). The SOVA instrument was composed of radiometers measuring the solar constant and two sets of IPHIRlike sunphotometers looking at the solar oscillations at wavelengths of 330 , 
$480,500,545$ and $865 \mathrm{~nm}$. The photometers have been observing the Sun for 9 months with a duty cycle of $60 \%$. That is to say with about the same duration as ACRIM but with a better duty cycle. Unfortunately, the temperature stabilization of the SOVA instrument introduced in the photometer signals spurious periods of the order of several minutes avoiding the detection of many $\mathrm{p}$ modes.

\subsubsection{Scientific outcomes}

With a time series of 7 months it was possible to detect only the strongest p modes of degree $l=0,1$ in the frequency range from 2.9 to $3.5 \mathrm{mHz}$ with a signal-to-noise ratio close to what it was obtained with ACRIM.

\subsection{DIFOS}

The last experiment of this history of space helioseismology to be launched was DIFOS on March 2,1994 aboard the CORONAS-I satellite (Gurtovenko et al., 1994). This satellite is still flying, its orbit is a circular quasi-synchronous Earth orbit with an altitude of $500 \mathrm{~km}$ and an inclination of 82.5 degrees. This kind of orbit allows continuous observation of the Sun during 20 days each 3 months, the remaining time consisting in orbit of $95 \mathrm{mn}$ with $35 \mathrm{mn}$ night. The DIFOS instrument is composed of a silicon photodiode detector with 2 glass filters at 550 and $750 \mathrm{~nm}$ and also a neutral glass leading to a bandwith of $400-1100 \mathrm{~nm}$ which is the spectral sensitivity range of the detector. DIFOS has been working until the $7^{\text {th }}$ of May 1994 when it broke down just before the satellite entered the period of 20 days of continuous observations. The time series obtained is therefore long of 50 days with a duty cycle of $65 \%$ (Lebedev et al, 1995).

\subsubsection{Scientific outcomes}

In a frequency range spanning from 2.2 to $3.8 \mathrm{mHz}$ were detected $\mathrm{p}$ modes of degrees $l=0,1$ and 2 . The signal-to-noise ratio better than 10 yielded to measurement of frequencies with an accuracy of $0.4 \mu \mathrm{Hz}$. As with IPHIR, it was also possible to follow the time-dependence of the modes and see the large variations of their amplitudes with time (Hasler et al., 1996).

\section{Present of space helioseismology}

The present of space helioseismology is fruitful because at the same time are flying three complementary experiments of helioseismology aboard the SOHO satellite (Domingo et al., 1995). SOHO (SOlar and Heliospheric Observatory), the solar mission of ESA and NASA, was successfully launched on December 2, 1995 and placed into halo orbit around the Earth-Sun lagrangian point L1. This mission should last three years at least and there is 
enough fuel in the spacecraft for many years. It is useful to briefly remind here the historical development of SOHO

\subsection{HISTORY OF SOHO}

In the context of helioseismology at the beginning of the eighties (see section 2 ) it was obvious that flying an experiment of helioseismology could greatly improve our knowledge of the solar structure. For this reason, in $1981 \mathrm{P}$. Delache proposed helioseismology as one of the science objectives of the potential ESA space mission DISCO (Dual Irradiance and Solar Constant Observations) during its assessment study.

One year later in November 1982 was proposed the SOHO mission in response to a call for mission proposals by ESA. Originally SOHO meant SOlar High-resolution Observatory and was dedicated to high-resolution spectroscopic investigation of the upper solar atmosphere. But, during the assessment study of SOHO in 1983 helioseismology was included in the scientific objectives of SOHO because DISCO was not being implemented.

Meanwhile in the United States was commissioned two SWG (science working group) one in 1978 to discuss what will be the best mission to follow the SMM mission and the other one in 1983 to consider the possibility of a space mission in helioseismology. Therefore already in 1983 thanks to their common goals, ESA and NASA envisaged to collaborate in SOHO.

For this reason the Science Study Team responsible of the Phase A study of SOHO was composed both of European and US scientists supported by ESA and NASA. Finally in February 1986 the Science programme Committee of ESA approved the STSP (Solar Terrestrial Science Programme) as the first cornerstone of the ESA's "Horizon 2000 programme". SOHO being one of the two components of the STSP with CLUSTER.

In March 1987 ESA and NASA made an Announcement of Opportunity for the STSP missions. This call for proposals led one year later to the selection of 12 experiments for SOHO which are shown here in a sketch of the satellite (see fig. 4) . Three of these experiments are dedicated to helioseismology : VIRGO, GOLF and SOI-MDI.

\subsection{VIRGO}

VIRGO (Variability of solar IRradiance and Gravity Oscillations) is an experiment which aims at determine the characteristics of low-degree solar $p$ modes and $\mathrm{g}$ modes measuring spectral and total irradiance changes (Fröhlich et al.,1995 , Fröhlich et al., these proceedings). VIRGO is composed of four different instruments :

- two different kinds of radiometers (PMO6-V and DIARAD),

- an IPHIR-like triple sunphotometer with interference filters $(402,500$ and 


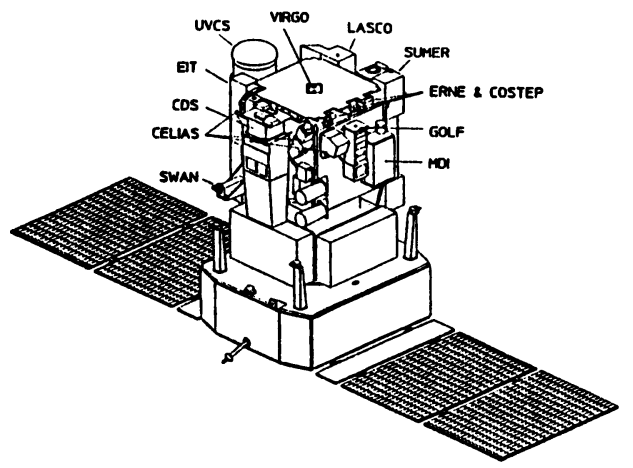

Figure 4. SOHO satellite (Domingo et al., 1995)

$862 \mathrm{~nm})$ and a Si-diode detector allowing detection of modes up to degree $l=3$,

- a Luminosity Oscillation Imager (LOI) based on a small Ritchey-Chretien telescope with a 16-pixel deep diffused silicon photodiode allowing detection of modes up to degree $l=7$.

\subsection{GOLF}

GOLF (Global Oscillations at Low Frequency) has also for scientific objectives to determine the characteristics of low-degree solar $\mathrm{p}$ modes and $\mathrm{g}$ modes. The complementarity with VIRGO is that GOLF measures variations of the integrated Doppler velocity on the solar disk (Gabriel et al., 1995 , Gabriel et al., these proceedings) instead of integrated sunlight variations. The GOLF instrument is based on an optical resonance technique using a sodium-vapour resonant scattering cell which allows to measure small Doppler shifts of the solar Na D1 and D2 lines. This technique is similar to the technique carried out in the IRIS network. (Grec et al., 1991)

\subsection{SOI-MDI}

SOI (Solar Oscillations Investigation) is an experiment which aims at observe intermediate and high degree modes (Scherrer et al., 1995, Scherrer et al. these proceedings) and is therefore complementary to VIRGO and GOLF. The scientific objectives are mainly to determine the characteristics of intermediate and high-degree solar p modes, detection and identification of $g$ modes, study of the convective zone dynamics and of magnetic structures. The instrument is a MDI (Michelson Doppler Imager) which is a kind of modified Fourier Tachometer tuned across the Ni line $(676.8 \mathrm{~nm})$. The detector is a $1024 \times 1024$ CCD camera with 2 resolutions: a low resolution of 


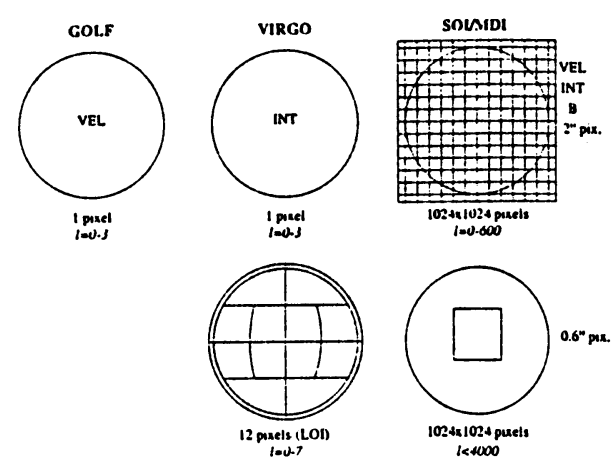

Figure 5. Comparison of GOLF-VIRGO and SOI-MDI kind of observations and degree coverage (Domingo et al., 1995)

2 " per pixel and a high resolution of 0.6 " per pixel. SOI produces various observables: Doppler velocity images, $\mathrm{Ni}$ line and continuum intensity,lineof-sight magnetic field, transverse velocity as well as limb position.

In Fig. 5 is summarized the type of observations done by each instruments and the corresponding degree coverage showing the complementarity of VIRGO-GOLF and SOI-MDI. These three instruments are now working well since more than 6 months. Their on-flight performances as well as the first results obtained with these ones can be found in these proceedings.

\section{Co-ordination of SOHO experiments with networks and other solar observations}

As shown in the previous section the three experiments of helioseismology aboard SOHO are complementary. In terms of mode degree the coverage of VIRGO, GOLF and SOI-MDI is from $l=0$ to about 4000 though above 1500 the overlap of the lines makes determination of frequencies difficult. The degree coverage is important because the more large it is the more accurate is the inference of the solar structure. In addition to degree coverage, time coverage is also very important. We know that p-mode frequencies are changing with the solar cycle (Pallé et al (1995)). It is therefore necessary to observe solar oscillations on timescale of the order of the solar cycle to better understand the physics of $p$ modes. This has already been done with the BISON and the IRIS networks which are working since the beginning of the eighties. In figure 5 is a plot of the degree coverage vs time coverage of the SOHO experiments and the ground-based networks. It shows how SOHO and the networks are complementary from the point of view of time coverage and degree coverage. Moreover, both velocity and irradiance observations are represented among these experiments. Helioseismology has 


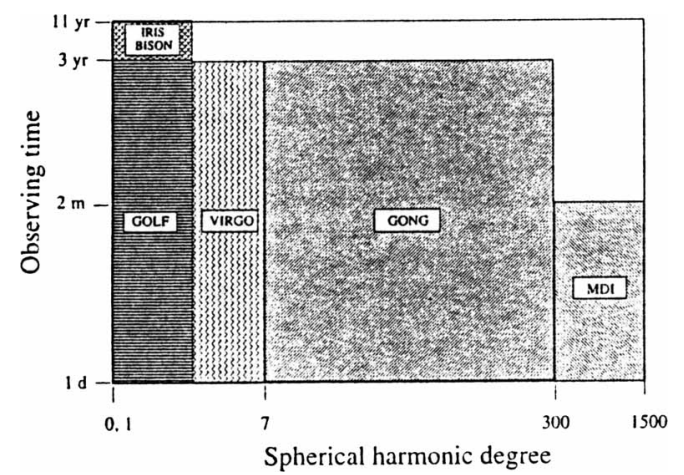

Figure 6. time coverage vs degree coverage of ground-based networks and SOHO experiments

never been so strong in terms of instruments observing at the same time the same oscillations from ground and from space in velocity and in intensity. It is a good opportunity to combine these different data sets together or with other solar observations in order to reinforce their impact on the inference of the solar structure and rotation.

\subsection{CO-ORDINATION WITH SYNOPTIC PROGRAMMES}

It well-known that experiments looking at oscillations in the Doppler shift of lines (like GOLF) are very sensitive to the magnetic activity on the solar surface (Ulrich et al.,1991). In this case it is useful to get informations from the synoptic programmes on ground to correct the line shape deformation due to magnetic effects. This will tend to reduce the solar noise in the power spectra and consequently to improve the detection of modes of low amplitude. These synoptic programmes are also useful because they provide helioseismologists with various indexes of activity which can be compared to the time-dependence of the solar oscillations parameters (frequency, amplitude) in order to better understand how p modes are sensitive to solar activity.

\subsection{CORRELATION OF TIME SERIES}

It is very unlikely that any of the experiments of helioseismology is free of non solar noise. With a time series of only one instrument it is difficult to distinguish between noise of solar origine and other noises. Therefore it is important to compare equivalent time series of different experiments doing the same kind of observations to detect any differences which could be explained in terms of non solar noise. Moreover correlation of time series 
will certainly improve the detection of the low amplitude modes (low order $\mathrm{p}$ modes and $\mathrm{g}$ modes). A third application of correlation of time series is the correlation of velocity signal with intensity signal (see e.g. Jiménez, 1994). The phase lag between signals and their amplitude ratio help us to study the nonadiabatic effects which are taking place in the photosphere.

\subsection{COMPARISON OF MODE PARAMETERS}

Once the mode parameters have been obtained from the time series it is also useful to compare them among the different experiments to check their consistency and detect any systematic errors, a task which is impossible with only one set of parameters. For example, from the existing sets of low-degree p-mode frequencies we can see that for some modes the values obtained with various experiments do not agree within the given error bars. Thus, either there are systematic errors or frequencies are changing on time scale quite smaller than the solar cycle. In such a case it is important to work on similar time series from different sources to understand where the discrepancies come from: systematic errors or unaccurate model of the physics of $p$ modes? These differences are even larger in the case of lowdegree p-mode splittings, showing how much work still remains to do as far as comparisons are concerned.

\subsection{MERGING OF FREQUENCY OR SPLITTING SETS}

With SOHO experiments and ground-based networks, the number of datasets which are or will be soon available has never been so large as well as the degree coverage. Assuming that frequency or splitting sets have been corrected from any systematic errors or that our model of mode physics has been improved their merging and their inversion will greatly extend our knowledge of the Sun's structure and its rotation.

\section{Future and prospects}

The next future of space helioseismology missions is of course still based on SOHO which is maintained until 1998 at least. Nevertheless, keeping SOHO observing all along the coming solar cycle could greatly improve the scientific outputs of the 12 embarked experiments.

It is also planned by Russia to fly in the next years two more CORONAS satellites (Oraevsky and Fomichev, 1996). On the first one (CORONAS-F) will be embarked a new DIFOS experiment. This one is slightly different from the original one described in section 2.5 , the reason is that the new instrument is based on a 6-channel sunphotometer instead of a 3-channel sunphotometer. 


\section{References}

Appourchaux, T., Toutain, T., Jiménez, A., Rabello Soares M.C., Andersen, B.N., Jones, A.R., (1995) Results from the luminosity oscillations imager, ESA-SP publications, Vol. 376,pp. 265

Baudin, F., Gabriel, A., Gibert, D., Pallé, P.L., Régulo, C., (1996) Temporal characteristics of solar p-modes, Astron. Astrophys.,Vol. 311,pp. 1024

Claverie, A., Isaak, G.R., McLeod, C.P., van der Raay, H.B., Roca Cortes, T., (1979) Solar structure form global studies of the 5-minute oscillation, Nature,Vol. 282,pp. 591

Crommelynck, D., Fichot, A., Lee, R.B., III; Romero, J.,(1995) First realisation of the space absolute radiometric reference (SARR) during the ATLAS-2 flight period, Advances in Space Research, Vol. 16, pp 17

Deubner, F.L., (1975) Observations of low wavenumber nonradial eigenmodes of the Sun, Astron. Astrophys., Vol. 44,pp. 371

Domingo, V., Fleck, B., Poland, A.I., (1995) The SOHO mission: an overview, Sol.Phys., Vol. 162,pp. 1

Fröhlich, C., (1984) Wavelength dependence of solar luminosity fluctuations in the five minutes range, Mem. S.A.It., Vol. 55,pp. 237

Fröhlich, C., van der Raay, H.B., (1984) Global solar oscillations in irradiance and velocity: A comparison, ESA The Hydromagnetics of the Sun,pp. 17

Fröhlich, C., Bonnet, R.M., Bruns, A.V., Delaboudinière, J.P., Domingo, V., Kotov, V.A.; Kollath, Z.; Rashkovsky, D.N., Toutain, T., Vial, J.C., (1988) IPHIR: the helioseismology experiment on the PHOBOS mission, ESA Publications, SP-286,pp. 359

Fröhlich, C., Romero, J., Roth, H., Wehrli, C., Andersen, B.N., Appourchaux, T., Domingo, V., Telljohann, U., Berthomieu, G., Delache, P., Provost, J., Toutain, T., Crommelynck, D., Chevalier, A., Fichot, A., Däppen, W., Gough, D., Hoeksema, T., Jiménez, A., Gómez, M.F., Herreros, J.M., Roca cortés, T., Jones, A.R., Pap, J.M., Willson, R.C., (1995) VIRGO: Experiment for helioseismology and solar irradiance monitoring,Sol.Phys., Vol. 162,pp. 101

Gabriel, A.H., Grec, G., Charra, J., Robillot, J.-M., Roca cortés, T., Turck-chièze, S., Bocchia, R., Boumier, P., Cantin, M., Cespédes, E., Cougrand, B., Crétolle, J., Damé, L., Decaudin, M., Delache, P., Denis, N., Duc, R., Dzitko, H., Fossat, E., Fourmond, J.-J., Garcia, R.A., Gough, D., Grivel, C., Herreros, J.M., Lagardère, H., Moalic, J.-P., Pallé, P.L., Pétrou, N., Sanchez, M., Ulrich, R., van der Raay, H.B., (1995) Global oscillations at low frequency from the SOHO mission (GOLF), Sol.Phys., Vol. 162,pp. 61

Grec, G., Fossat, E., Pomerantz, M., (1980) Solar oscillations: Full disk observations from the geographic south pole, Nature, Vol. 288,pp. 541

Grec, G., Fossat, E., Gelly, B., Schmider, F.X., (1991) ,Sol. Phys., Vol. 133,pp. 13

Gurtovenko, E.A., Kesel'man, I.G., Kostyk, R.I., Osipov, S.N., Lebedev, N.I., Kopayev, I.M., Oraevsky, V.N., Zhugzhda, Yu.D., (1994) Photometer "DIFOS" for the study of solar brightness variations, Sol.Phys., Vol. 152, pp. 43

Hasler, K.-H., Zhugzhda, Yu.D., Lebedev, N.I., Arlt, R., (1996) Observation of solar low-I p-modes by the CORONAS-DIFOS experiment, to appear in Astron. Astrophys.

Innocenti, L., (1993), EURECA-1 Science operations, ESA mission report

Jiménez, A., (1994) Phase differences between irradiance and velocity low degree solar acoustic modes revisited, Sol. Phys., Vol. 152 no. 1, pp. 319

Lebedev, N.I., Oraevsky, V.N., Zhugzhda, Yu.D., Kopayev, I.M., Kostyk, R.I., Pflug, K., Rüdiger, G., Staude, J., Bettac, H.-D., (1995) First results of the CORONAS-DIFOS experiment, Astron. Astrophys., Vol. 296,pp. L25

Leibacher, J.W., Stein, R.F., (1971) A new description of the five-minute solar oscillation, Astrophys. lett.,Vol. 162,pp. 191

Leighton, R.B., Noyes, R.W., Simon,G.W., (1960) Aerodynamic phenomena in stellar atmosphere, Suppl. Nuovo Cimento, Vol. 22, pp. 321

Noyes, R.W., Rhodes Jr., E.J.,(1984) Probing the depths of a star: the study of solar 
oscillations from space, $N A S A$ report

Oraevsky, V.N., Fomichev, V.V., (1996) Russian future projects, $A d S p R$, Vol. 17 no. 4,5 ,pp. 359

Pallé, P.L.,(1996) The state of art on helioseismic ground-based experiments, These proceedings

Pallé, P.L.,(1995) Solar cycle frequency shifts at low l, ASP Conf. series, Vol. 76, pp. 239

Scherrer, P.H., Bogart, R.S., Bush, R.I., Hoeksema, J.T., Kosovichev, A.G., Schou, J., Rosenberg, W., Springer, L., Tarbell, T.D., Title, A., Wolfson, C.J., Zayer, I., MDI engineering team, (1995) The solar oscillations investigation-Michelson Doppler imager, Sol.Phys., Vol. 162,pp. 129

Toutain, T., Fröhlich, C.,(1992) Characteristics of solar p-modes: results from the IPHIR experiment, Astron. Astrophys.,Vol. 257, pp. 287

Toutain, T., Kosovichev, A.G., (1994) Anew estimate of the solar core rotation from IPHIR, Astron. Astrophys., Vol. 284,pp. 265

Ulrich, R.K. (1970) The five-minute oscillations on the solar surface, Astrophys. J., Vol. 162,pp. 993

Ulrich, R.K. (1991) A co-ordinated and synergistic analysis strategy for future gorundbased and space helioseismology, $A d S p R$,Vol. 11 no. 4,pp. 217

Willson, R.C., (1979) Active cavity radiometer type IV, Appl. Opt., Vol. 18,pp. 179

Woodard, M., (1984) Short-period oscillations in the total solar irradiance, Phd Thesis, University of California

Woodard, M., Hudson, H., (1983) Solar oscillations observed in the total irradiance, Sol. Phys., Vol. 82,pp. 67

Woodard, M., Hudson, H., (1983),Frequencies, amplitudes and linewidths of solar oscillations from total irradiance observations, Nature, Vol. 305,pp. 589 